
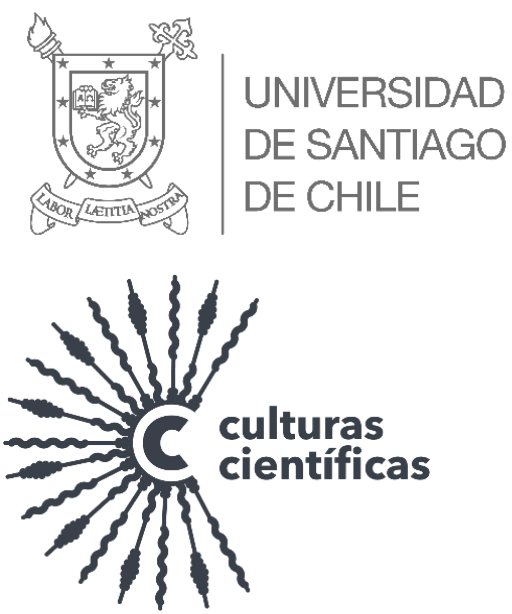

Franco Bastián Menares Paredes

fmenaresp@gmail.com

Universidad de Chile

https://orcid.org/0000-0002-5959-8209

Artículo recibido: 08 de septiembre de 2021

Artículo aceptado: 30 de septiembre de 2021

Artículo publicado: 30 de diciembre de 2021

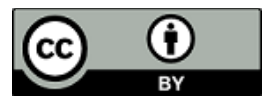

Artículo de Investigación https://doi.org/10.35588/cc.v2i2.5101

\section{Una objeción pluralista al argumento de los milagros}

\author{
A Pluralist Objection Against the No-Miracle Argument
}

\section{Resumen}

El objetivo de este artículo es elaborar una objeción en contra del argumento realista conocido como 'argumento de los milagros' (NMA) en el debate del realismo científico. El NMA se basa en la concepción de que el realismo científico es la filosofía que mejor explica el éxito de las ciencias. Se objeta que, si tomamos en serio las consideraciones del pluralismo científico, no existe una noción unívoca de éxito a la cual el argumento pueda apelar. De esto se sigue que o bien no es posible inferir verdad a partir del éxito de una teoría o bien que debemos aceptar que las ciencias nos ofrecen múltiples verdades acerca de un mismo dominio de investigación. Frente a esto, se ofrecen tres soluciones: primero, intentar salvar la validez del NMA ofreciendo una alternativa que permita evadir las dificultades planteadas. Tal alternativa, sin embargo, no se encuentra disponible en la literatura. Segundo, reconceptualizar la noción de «verdad» o asumir el pluralismo metafísico, lo que nos llevaría a asumir posturas consideradas contraintuitivas. Por último, dispensar el NMA del debate del realismo científico y asumir un empirismo pluralista que sea capaz de dar cuenta de la pluralidad de las ciencias sin comprometerse con nociones alternativas del concepto de «verdad» o con interpretaciones metafísicas del pluralismo.

Palabras clave: Realismo científico, Pluralismo Científico, Inferencia a la mejor explicación, Empirismo.

\begin{abstract}
The aim of this article is to elaborate an objection against the realist argument that, in the debate on scientific realism, is known as the 'No-Miracles Argument' (NMA). This argument hinges on the assumption that scientific realism is the philosophy that best explains the success of science. Here, it is objected that if the considerations from scientific pluralism are to be taken seriously, there is no univocal conception of «success» at hand. From this it follows that either we are not able to infer theory's «truth» from theory's «success», or that we must accept that science offers several truths about the same inquiry's domain. I outline three solutions to face this issue: first, to defend the NMA against the pluralist's objections, nevertheless there is no account in the literature that can meet these objections. Second, either to reconceptualize the notion of «truth» or embrace a metaphysical pluralism, yet these accounts are highly counterintuitive. Finally, to dispense the NMA from scientific realism debate, and, instead, to assume an empiricist pluralism that can account for sciences' plurality without a compromise with alternative notions of «truth» or metaphysical interpretations of scientific pluralism.

Keywords: Scientific realism, Scientific pluralism, Inference to the best explanation, Empiricism.
\end{abstract}




\section{Introducción}

En el debate sobre realismo científico, el argumento favorito de los realistas ha sido el «argumento del milagro» (NMA, por sus siglas en inglés), el cual consiste en plantear que la mejor manera que tenemos para explicar el éxito empírico de las ciencias es asumiendo que sus teorías son aproximadamente verdaderas (Putnam 1979; Psillos 1999). Chang (2017), sin embargo, argumentando a favor de la necesidad de que el realista adquiera un compromiso pluralista, nos muestra cómo de la constatación de la diversidad de metas y objetivos que guían la práctica científica en sus diversas ramas se infiere la necesidad de reconsiderar la noción de éxito en el contexto de las ciencias. Esta revisión pone en aprietos al realista que busca inferir la verdad a partir del éxito de las teorías, pues veremos que no contamos con una manera única e incontrovertible de medir el éxito de ellas. De este modo, veremos que aún asumiendo la cuestionada premisa realista de que el éxito es conducente a la verdad, las consecuencias que se extraen de este argumento son indeseables para el realista.

En el presente trabajo me propondré mostrar cómo, si nos tomamos en serio la reconsideración pluralista de la noción de éxito en el contexto de la pluralidad de las prácticas científicas, el NMA se hace insostenible al menos para defender alguna versión estándar de realismo científico. Para ello, en la sección 2, expondré el NMA. Luego, en la sección 3 expondré las consideraciones que llevan al pluralista a cuestionar la noción monista de éxito empírico y mostraré que, de aceptar la concepción pluralista de éxito, del NMA se siguen consecuencias que entran en contradicción con los compromisos que usualmente asume el realista. Finalmente, en la sección 4 evaluaré tres salidas que admite el problema: (a) defender la validez del NMA y ofrecer una cuenta unificada de la concepción de éxito en ciencias, (b) ofrecer una recaracterización del realismo que nos permita lidiar con estas consecuencias y (c) rechazar la validez del NMA.

\section{Argumentando por el realismo científico}

Por realismo científico se entiende toda doctrina que asume que nuestra ciencia (o una parte de ella) nos entrega descripciones verdaderas del mundo (tanto observable como inobservable). Desde el sentido común — prefilosófico_- el realismo se muestra como una actitud intuitiva y natural: el prestigio del que goza la ciencia, por haber demostrado ser nuestro mejor método o conjunto de prácticas para producir conocimiento, está ampliamente extendido y puede llevar a quien no se detenga a pensarlo cuidadosamente a asumir una actitud acrítica con respecto a los límites del conocimiento que nos pueden entregar nuestras prácticas científicas e ignorar los problemas que surgen del examen de éstas. La realidad es que el problema del realismo científico es un problema genuino, y del que no se ha escrito poco. Al contrario, ha estado en buena medida en el centro del debate de la filosofía de las ciencias durante las últimas décadas.

Desde un punto de vista filosófico, la principal motivación para asumir el realismo reside en el abrumador éxito que reportan nuestras ciencias a la hora de ofrecer explicaciones, manipular y predecir diversos fenómenos. Entre los ejemplos que podemos ofrecer para ilustrar el éxito empírico que suele reportar la ciencia se cuentan el descubrimiento de nuevos planetas por medio de 
Franco Bastián

Menares Paredes

inferencias guiadas por las teorías físicas de Newton $^{1}$ o la capacidad de manipular sistemas infinitamente complejos y distantes de la experiencia humana en contextos experimentales, como ocurre en el bombardeo de positrones y electrones que se ejecuta para controlar la carga eléctrica de un objetivo relevante ${ }^{2}$. Este fascinante hecho ha llevado a muchos filósofos y filósofas a preguntarse por la explicación de este éxito, y no pocos han llegado a la conclusión de que la razón que lo explica no puede ser otra sino que la ciencia es capaz de penetrar en el mundo y revelarnos cuáles son las entidades y leyes fundamentales que dan forma a estos fenómenos.

Esta última intuición es la que intenta ser recogida en el principal argumento esgrimido por el realismo a la hora de justificar su optimismo epistémico. Este argumento es comúnmente conocido como el argumento de los no milagros [No Miracles Argument], o, dicho de manera más simple, argumento del milagro. En palabras de Hilary Putnam, el argumento versa de la siguiente manera:

El argumento positivo para el realismo es que es la única filosofía que no hace del éxito de las ciencias un milagro. Que los términos en las teorías científicas maduras típicamente refieren (acorde con la formulación de Richard Boyl), que las teorías aceptadas en ciencias maduras son típica y aproximadamente verdaderas, y que un mismo término puede referir a lo mismo incluso cuando ocurre en teorías distintas, todos estos enunciados no son considerados como verdades necesarias, sino como parte de la única explicación científica del éxito de las ciencias, y, por lo tanto, como parte de cualquier descripción adecuada de las ciencias y de su relación con sus objetos (Putnam, 1979$, p. 73$)^{3}$.

Lo que expresa este argumento, a grandes rasgos, puede parafrasearse de la siguiente manera: ante el hecho del exitoso desempeño de nuestras teorías científicas, la mejor explicación que podemos ofrecer de este fenómeno es que generalmente ellas son (al menos) aproximadamente verdaderas. En consecuencia, la aceptación de una teoría conllevaría el creer que ella es (al menos) aproximadamente verdadera.

Esta formulación general del NMA ha sido, sin embargo, refinada y matizada de diversas maneras, engendrando una familia de argumentos que atienden a diversos aspectos del debate del realismo científico ${ }^{4}$. El matiz del argumento que nos concierne es el que busca aclarar qué debemos entender por éxito en la enunciación de este argumento. No obstante, la respuesta que podamos dar depende de la postura que asumamos respecto al sentido que le otorgamos a la noción de éxito cuando hablamos en el contexto de las ciencias en general.

\footnotetext{
${ }^{1}$ Un ejemplo histórico recurrente es el de la predicción de la existencia de un planeta no observado hasta ese momento que hubiera explicado ciertas perturbaciones en la órbita de Urano con respecto a los cálculos que se hacían en base a las teorías de Newton. Dicho planeta pudo ser finalmente observado según los cálculos ofrecidos, y resultó ser el planeta que hoy conocemos como Neptuno.

2 Este ejemplo es ofrecido y descrito en Hacking (1983, p. 23)

${ }^{3}$ N. del. Ed.: todas las traducciones fueron realizadas por el autor del artículo.

${ }^{4}$ Para una visión general sobre los desarrollos posteriores del NMA se sugiere consultar Borge (2015)
} 
Una objeción pluralista

al argumento de los milagros

No cualquier clase de éxito funciona para argumentar la posición realista. Un tema clásico en filosofía de las ciencias es el que trata sobre la diferencia entre aquellas teorías que son capaces de ofrecer novedad [novelty] (predicciones novedosas), y aquellas que se limitan a dar cuenta de fenómenos que ya son conocidos en al momento de su formulación. En este contexto, que una teoría sea capaz de ofrecer una buena explicación de aquellos fenómenos para cuya explicación la teoría fue formulada no es un hecho que necesariamente debiera sorprendernos, y no constituye una buena razón para sostener que dicha teoría explica en virtud de que ella es verdadera. En cambio, una teoría que es capaz de ofrecer predicciones novedosas sí posee una robustez epistémica que la hace una buena candidata para sostener el argumento de que ella es capaz de ofrecer dichas predicciones en virtud de que es (al menos) aproximadamente verdadera. Esta última es la razón por la cual los realistas suelen distinguir entre teorías maduras y aquellas que no lo son, y al éxito genuino de aquel que sería sólo aparente. En consecuencia, vemos que los realistas suelen cualificar la noción de éxito a la hora de ofrecer su argumento restringiendo el tipo de éxito que es considerado al éxito empírico que pudieran exhibir las teorías.

En un espíritu similar a la intuición desarrollada en el párrafo anterior, los realistas suelen distinguir entre aquellos elementos de las teorías que se conservan a través de los distintos cambios teóricos y aquellos elementos que con el tiempo son desechados y dejan de ser considerados por los científicos como conceptos válidos para describir el mundo. Ejemplos de esto son conceptos como los de flogisto o éter calórico. Psillos (1999) llama a esto el movimiento «divide et impera». La consecuencia más relevante que esta consideración tiene para el NMA es que debemos considerar que los elementos que explican el éxito empírico de las teorías no son la totalidad de las entidades y leyes que ella postula, sino sólo aquellas que tiene un rol indispensable en el éxito de esta (Psillos, Ibid., p.104).

Por otro lado, conviene observar que los realistas no consideran que las virtudes epistémicas que nos llevan a la verdad de las teorías sean agotadas por su dimensión empírica. Putnam, en el extracto anteriormente citado, habla de la postulación de la verdad aproximada de las teorías como la única explicación científica de su éxito. Pero ¿en qué sentido es esta explicación científica? En ciencias ocurre que en ciertas ocasiones tenemos teorías rivales que son respaldadas en igual medida por los datos empíricos que disponemos; en esos casos se hace patente que al momento de evaluar nuestras teorías también entran en juego consideraciones extra-empíricas. Una de ellas -quizá la más importante - es el poder explicativo que posee cada teoría. En analogía a como ocurre en ciencias, Putnam postula que el realismo debe ser considerado verdadero por ser, de las opciones disponibles, la filosofía que ofrece la mejor explicación del éxito de las ciencias, es decir, por su poder explicativo. Este razonamiento pertenece a una clase de inferencias conocidas como inferencias a la mejor explicación (IBE, por sus siglas en inglés), o inferencias abductivas. Estas últimas se caracterizan por fundar sus juicios en base a consideraciones explicativas: si determinada explicación es la mejor que tenemos disponible para dar cuenta de determinado fenómeno, entonces dicha explicación debe ser verdadera. La principal diferencia entre realistas y anti-realistas en el contexto del debate por el realismo científico es que, mientras que los realistas consideran el poder explicativo como una virtud epistémica y por lo tanto incorporan la IBE en su batería de inferencias, 
los anti-realistas consideran el poder explicativo como una virtud meramente pragmática $\mathrm{y}$, por lo tanto, no están dispuestos a comprometerse con la verdad de aquellas inferencias. ${ }^{5}$

Una de las consecuencias que puede ser extraída y generalizada a partir de la aplicación de la IBE al realismo científico es el siguiente precepto — ampliamente adoptado por los realistas: el éxito es conducente a la verdad [truth conducive]. Si aceptamos la premisa de que el éxito de una teoría puede explicarse en virtud de su proximidad a la verdad — tal como concluye el NMA—, estamos justificados en concebir el éxito de nuestras teorías como un indicador de la verdad (aproximada) de ellas. Puesto de otro modo, si adoptamos esta premisa - el éxito es conducente a la verdad-, entonces podemos inferir que allí donde nos encontremos con teorías exitosas estas serán (al menos) aproximadamente verdaderas.

Es preciso señalar que tanto las IBE, en general, como el NMA, en particular, despiertan escepticismo en una parte importante de la comunidad filosófica. Mencionaremos muy brevemente dos de las principales objeciones que se suelen plantear: en primer lugar, se acusa al NMA de caer en la falacia de la tasa base [base rate fallacy], la cual consiste, a grandes rasgos, en ignorar la información general acerca de la probabilidad de que determinada situación sea el caso y concentrase en la consideración de casos particulares. En el caso del NMA esto se aplica al hecho de que la inferencia se hace ignorando la tasa base de teorías aproximadamente verdaderas. En segundo lugar, se suele objetar que — como mencionábamos más arriba — las consideraciones de tipo explicativas no son de carácter epistémico sino de carácter meramente pragmático; por lo tanto, ellas no serían conducentes a la verdad. De esta manera, se argumenta que no estamos justificados en inferir la verdad a partir del éxito por la mera consideración de que lo primero explicaría lo segundo. Esta última inferencia es la que constituye el núcleo del NMA.

En todo caso, quizás la crítica más discutida en la literatura es la imputación de circularidad que se le hace al NMA. Se suele sostener que el NMA es viciosamente circular pues, a pesar de ser una instancia de IBE, requeriría a su vez una IBE para su fundamento. Psillos (1999, p.80 y ss.) argumenta que es necesario distinguir entre una circularidad al nivel de las proposiciones y una circularidad propia del meta-nivel de las reglas de inferencia. Según Psillos, si el NMA cae en algún tipo de circularidad, es en una circularidad de este segundo tipo. Lo anterior, el argumento sigue, no tiene por qué preocupar al defensor del NMA, pues todas las formas de razonamiento ampliativo, e incluso inferencias deductivas como el modus ponens, incurrirían de una u otra manera en una circularidad de reglas de inferencia. Ahora bien, el argumento anterior supone comprometerse en buena parte con un background epistemológico externalista que el o la anti-realista difícilmente estaría dispuesta a aceptar. Es por esto que, en un artículo posterior (Psillos, 2011b), el autor termina por deflacionar el alcance del argumento y defiende en cambio la tesis de que el NMA no debe ser

\footnotetext{
${ }^{5}$ Psillos (1999, p.75 y ss.) construye al NMA como una defensa explicacionista del realismo científico. Ante las objeciones mencionadas en este párrafo, Psillos propone asumir una epistemología externalista que implica que las reglas de inferencia no deben ser justificadas para ser utilizadas, sino más bien que ellas deben explicar y defender (en un sentido externalista) determinados métodos y prácticas inferenciales. Sin embargo, él mismo luego rectificaría (Psillos, 2011a) y propondría que se debe entender al NMA como justificando la fiabilidad de las IBE. Volveremos a este punto un par de párrafos más adelante cuando hablemos de la amenaza de circularidad a la que el NMA debe responder.
} 
Una objeción pluralista

al argumento de los milagros

interpretado como un argumento a favor del realismo científico, sino como un argumento que funciona dentro del marco del realismo científico para validar la IBE ${ }^{6}$.

En todo caso, veremos que no es necesario asumir alguno de estos criticismos para cuestionar la fortaleza del argumento realista, sino que éste presenta problemas incluso aceptando la cuestionable premisa de que el éxito es conducente a la verdad. En lo que sigue se mostrará cómo aún aceptando la validez de la IBE, el NMA presenta consecuencias indeseables para la concepción realista. La consideración relevante que debemos tener en vista es que existen diversas maneras de entender la noción de éxito en ciencias y filosofía de las ciencias. En la siguiente sección nos dedicaremos a examinar este concepto.

\section{La reconsideración pluralista de la noción de éxito en las ciencias}

Se conoce por el nombre de pluralismo científico a un emergente programa de investigación en filosofía de las ciencias que ha llamado la atención sobre muchos presupuestos que la visión recibida ${ }^{7}$ integraba acríticamente en su concepción de la ciencia. La concepción «monista» de la ciencia se caracteriza por concebir a la ciencia como una empresa epistémica unificada que compartiría un mismo método a lo largo y ancho de todas sus ramas. Esta tendría sus bases en la física, desde donde supervendrían todos los otros fenómenos estudiados por sus demás subdisciplinas; y que sería capaz de excluir toda interferencia de valores e intereses en su labor incorruptible por la búsqueda de la verdad. Esta concepción se constituyó como un motivo que por mucho tiempo acompañó las investigaciones de la comunidad científica y la reflexión de la comunidad filosófica, sobre todo en los albores de la filosofía de la ciencia. Sin embargo, con el surgimiento del movimiento pluralista esta visión comenzó a enfrentar críticas que cuestionaban la idoneidad de esta imagen científica por considerarse exageradamente idealizada y perdió su lugar hegemónico entre estas comunidades.

No es difícil notar que, de hecho, el ejercicio de las ciencias exhibe una buena cuota de desunidad o pluralidad. Las investigaciones en las que trabajan las y los economistas no se parecen mucho a las que se desarrollan en otras disciplinas como la geología, por poner un ejemplo. Así mismo, es más o menos evidente que los métodos de investigación empleados en física teórica tienen poco o nada que ver con aquellos que se utilizan en antropología. Incluso, dentro de una misma disciplina — como la biología - las prácticas científicas pueden llegar a ser tan diversas como aquellas propias de la zoología, por un lado, y aquellas asociadas a la biología molecular, por otro. Planteadas las cosas de esta manera, ¿hasta qué punto podemos afirmar que existe una unidad de

\footnotetext{
${ }^{6}$ Cabe mencionar que una consideración similar es presentada por John Worrall (2011), donde apunta a que el NMA debe considerarse más bien como una intuición que como un argumento propiamente tal.

${ }^{7}$ Se suele hablar de "visión" o "concepción" recibida para referirse a la concepción sintáctica de las teorías científicas. Aquí me permito utilizar la expresión en un sentido más general para hablar, no sólo de la visión sintáctica de las teorías, sino del conjunto de concepciones que inspiraron la corriente dominante en filosofía de las ciencias durante la primera mitad del siglo pasado.
} 
Franco Bastián

Menares Paredes

método, prácticas o valores epistémicos a lo largo de todo el rango de disciplinas que ubicamos bajo el ancho concepto de «ciencia»? Hasta hoy la comunidad filosófica no ha sido capaz de ponerse de acuerdo para dar una cuenta unificada y coherente de alguna clase de continuidad metodológica a lo largo de toda la diversidad de prácticas científicas que pudiera con justicia identificarse como el método científico; más bien, el consenso parece ser que no hay tal cosa como eso (HoyningenHuene, 2013). Asimismo, cuando se trata de dar cuenta de los criterios que las y los científicos emplean a la hora de elegir sus teorías, no es del todo simple y no-problemático identificar qué valores epistémicos son los que priman, ni de qué manera ellos interactúan en el proceso (Longino, 1996). El análisis del proceso de cómo las y los científicos elaboran sus teorías es aún más intrincado, y pareciera que el consenso es que definitivamente no se puede dar una cuenta racional de éste ${ }^{8}$.

En términos más concretos, el pluralismo científico ha sido caracterizado de muchas maneras: como una tesis metafísica acerca de la des-unidad fundamental del mundo (Dupré 1993; Cartwright, 1999); como una determinada actitud [stance] escéptica frente a ciertos proyectos de unificación de las ciencias (Kellert, Longino \& Waters, 2006); como una ideología que aboga por diversificar los programas de investigación en ciencias, y conservar aquellos que se consideran fueron vencidos (Chang, 2012); como una determinada concepción acerca de lo que conlleva la aceptación de una teoría (Glick, 2021); entre otras. A la luz de lo expuesto, podemos notar que el pluralismo es un movimiento diverso. Sin embargo, para efectos de este argumento no se asumiremos ninguna forma de pluralismo en particular. Nos bastará con quedarnos con la idea que inspira esta familia de aproximaciones: la ciencia es diversa, y esta diversidad no debe tratar de ser eliminada (en principio), sino que debe incorporarse en nuestra imagen de cómo ella funciona.

¿Qué nos dice el pluralismo respecto al concepto de éxito en las ciencias? Siguiendo el argumento de Chang (2017), lo primero que debemos notar es que el concepto de éxito es un término cuyo significado es en gran medida de carácter relativo: el éxito por lo general refiere a una determinada meta trazada por aquel agente del que se dice que tuvo éxito. Por lo tanto, para preguntarnos por el éxito de la ciencia, primero debemos preguntarnos por su(s) meta(s) u objetivo(s).

En primer lugar, una respuesta bastante intuitiva pudiera ser el sostener que el objetivo de las ciencias radica en ofrecer teorías verdaderas: decimos que lo que buscamos al formular teorías es ofrecer descripciones verdaderas acerca de cómo funciona el mundo. Decimos que una investigación ha alcanzado el éxito cuando ha logrado dar con una teoría o modelo que putativamente ofrece una caracterización verdadera de aquello que se ha propuesto investigar. Si el objetivo de la ciencia es ofrecer descripciones verdaderas acerca del mundo, entonces, se entiende que ella será exitosa cuando las descripciones que formule sean verdaderas. Pero ¿cómo sabemos que estas descripciones son verdaderas? Estas consideraciones nos llevan de nuevo al principio del asunto: en ausencia de un método independiente para confirmar la veracidad de nuestras teorías debemos ofrecer criterios alternativos que nos indiquen que nos encontramos frente a una teoría verdadera.

\footnotetext{
${ }^{8}$ Este es un tema clásico en filosofía de la ciencia que puede ser rastreado hasta el trabajo de Hans Reichenbach (1938)
} 
Una objeción pluralista

al argumento de los milagros

Una segunda opción —en principio bastante plausible - es postular que el objetivo de las ciencias es ofrecer explicaciones de los fenómenos que son objeto de su estudio. Entonces, según este enfoque una empresa científica será exitosa cuando sea capaz de ofrecernos una explicación satisfactoria de determinado conjunto de fenómenos que constituyen su dominio. Pero ¿es la capacidad de ofrecer explicaciones condición suficiente para considerar a una teoría exitosa? Se suele considerar que entre mayor poder o alcance explicativo tiene determinada teoría, menores son las probabilidades de que ella sea verdadera. Por lo tanto, pareciera que verdad y explicación serían objetivos que conflictuarían entre ellos. Esto último no tiene por qué necesariamente ser un problema, pero sí resulta problemático si pretendemos afirmar que el éxito es conducente a la verdad - lo que es el caso para el NMA. Además, existen buenas y malas explicaciones; esto último pudiera parecer trivial ya que nadie afirmaría que una teoría es exitosa si sólo nos ofrece malas explicaciones, y de ahí la exigencia de la cualidad de satisfactoria postulada al enunciar esta postura. Sin embargo, la dificultad resurge puesto que ahora nos enfrentamos con el desafío de ofrecer un criterio que nos permita diferenciar explicaciones satisfactorias de las que no lo son. Por lo tanto, el éxito de la ciencia no puede descansar meramente en su capacidad de ofrecer explicaciones.

Antes de tratar la última de las caracterizaciones tentativas del objetivo de la empresa científica, vale la pena dedicar unas palabras a la amenaza de circularidad que las interpretaciones anteriores suponen. El NMA postula que el éxito de las teorías se explica por su verdad. No obstante, es evidente que postular la verdad de las teorías como explicación de su éxito para luego caracterizar el éxito de las teorías en términos de la consecución de la verdad nos hace caer en una circularidad inadmisible. Es por esta razón que, a la luz de un análisis más cuidadoso, debemos descartar esta interpretación como válida para construir una defensa explicacionista del realismo científico ${ }^{9}$. De manera similar, si consideramos que el éxito de las ciencias consiste en ofrecer explicaciones satisfactorias, el argumento de que las teorías explican satisfactoriamente porque ellas son verdaderas se vuelve tautológico para el realista. Estas observaciones refieren a cuestiones estrechamente emparentadas con los asuntos que motivan las cualificaciones del NMA que presentamos en la sección anterior ${ }^{10}$.

Finalmente, examinemos la tercera y última interpretación. Podemos plantear que el objetivo de las ciencias es ofrecer teorías que sean empíricamente adecuadas. Según la concepción del empirismo constructivo (van Fraassen, 1980), la ciencia es una actividad que no está orientada al descubrimiento (de verdades acerca del mundo), sino que la actividad científica se centraría más bien en la construcción de modelos teóricos compatibles con aquellos modelos de fenómenos o datos que se extraen en el ámbito observacional o experimental: la ciencia busca la adecuación empírica de sus teorías. Pareciera que esto último tampoco estuviera tan alejado de la concepción realista que busca fundamentar su optimismo en el éxito empírico de las teorías. La diferencia, sin embargo, radica en que el éxito empírico para el realista se basa en la predicción de fenómenos novedosos tal como se mencionó al caracterizar la noción de «madurez» de las teorías. El empirista,

\footnotetext{
${ }^{9}$ Ver Psillos (1999, pp.78 y ss.) Como mencionábamos en la sección anterior, es necesario una construcción sofisticada del NMA para mitigar las amenazas de circularidad.

${ }^{10}$ Quisiera expresar mi gratitud con el revisor anónimo que me hizo reflexionar sobre este asunto.
} 
Franco Bastián

Menares Paredes

en cambio, no considera que la capacidad de ofrecer predicciones novedosas sea una característica epistémicamente relevante de las teorías, sino que es un elemento parasitario de su adecuación empírica (Bueno, 1999).

Independiente de lo anterior, esta imagen empirista de la ciencia tampoco es capaz de ofrecernos una respuesta satisfactoria al problema en cuestión. Según Chang (2017), la misma noción de adecuación empírica es susceptible de múltiples caracterizaciones, pues presenta una pluralidad de dimensiones y grados en los que ella puede ser alcanzada. Chang (2012. p.117) argumenta que podemos interpretar aquello que Kuhn (1962) identificaba como las virtudes epistémicas básicas que todos las y los científicos comparten, como encarnando, de hecho, distintas dimensiones de éxito empírico: la precisión expresaría la idea básica de calce empírico [empirical fit]; la consistencia de una teoría debe ser considerada no solo respecto a ella misma, sino también respecto a otras teorías aceptadas sincrónicamente (al mismo tiempo $t$ ), cada una de ellas con sus propias exigencias de éxito empírico; el alcance de una teoría señala la cantidad y variedad de observaciones de las que una teoría puede dar cuenta. Además — siguiendo con las virtudes kuhnianas — debemos considerar la fecundidad [fruitfulness] y resiliencia de las teorías en la evaluación de su éxito empírico. Después de todo, una teoría debe ser evaluada no solo respecto un determinado tiempo $t$, sino a su desempeño diacrónico a lo largo de la historia de la ciencia (tanto de su desempeño pasado como de su porvenir). Es el caso que teorías y modelos son muy exitosos a la hora de hacer predicciones, pero tienen poco sustrato empírico en su formulación (esto ocurre con notable frecuencia en las ciencias económicas), o bien — como nos muestra Nancy Cartwright (1983)— hay parcelas de teorización científica increíblemente resilientes al paso del tiempo y de las revoluciones científicas, pero con un alcance y aplicación extremadamente limitado ¿Cómo evaluar en estos casos el éxito empírico?

Pareciera que ninguna de las propuestas mencionadas pudiera dar una cuenta satisfactoria y completa del objetivo que persiguen las prácticas científicas $\mathrm{y}$, en consecuencia, no podemos caracterizar el éxito a partir de ellas. Cuando analizamos cómo opera en la práctica la noción de éxito en las ciencias, podemos observar que es posible articular diferentes nociones de éxito a partir de todo el espectro de virtudes epistémicas que sostenemos. A esto debemos también agregar el conjunto de virtudes pragmáticas que suelen ser valoradas en las teorías. Algunas de ellas son: elegancia, alcance, simplicidad, poder explicativo, poder unificador. En la misma línea, debemos que constatar que el concepto de éxito difícilmente se deja formular de tal manera que nos entregue un límite claro a partir de lo cual a alguien o algo se le considera exitoso. Éxito, más bien, es una cuestión de grados. Un concepto continuo y no uno discreto. ¿Qué tan exitosa debe ser una teoría para considerarla conducente a la verdad? Parece difícil ofrecer un criterio que satisfaga tal exigencia.

Se podría objetar que, aunque no haya un límite definido para separar aquellas teorías exitosas de las que no lo son, de igual manera sigue habiendo teorías más exitosas que otras. Esto, sin embargo, choca con la consideración de que el éxito nunca es uni-axial (o unidimensional), sino que siempre es evaluado de manera pluri-axial (o multidimensional). En otras palabras, a la hora de juzgar el éxito de determinada teoría nos vemos en la necesidad de ponderar una multiplicidad de factores de tal manera que no es posible ofrecer una jerarquización unívoca donde se sucedan las 
Una objeción pluralista

al argumento de los milagros

teorías según sus grados de éxito empírico. Cada valor epistémico, cada dimensión de éxito empírico, podría eventualmente ser considerada por encima de las otras y no es claro cómo ofrecer un criterio común por el cual juzgar el asunto (Chang, 2012).

No es un hecho muy difícil de observar que dentro de la comunidad científica —e incluso para un mismo científico o científica individual — lo que guía la investigación y la aceptación de teorías son una pluralidad de valores y virtudes tanto epistémicas como pragmáticas ${ }^{11}$. Al igual que en cuestiones de método, como mencionábamos más arriba, en cuestiones de metas u objetivos es imposible reducir o subsumir todas estas virtudes bajo un mismo concepto que las abarque a todas y nos señale en términos unitarios qué es aquello que las ciencias se esfuerzan por conseguir.

Dijimos que el NMA es el principal argumento que los realistas emplean para justificar su optimismo respecto a la ciencia. Los realistas suelen sostener que a pesar de que no podemos acceder de modo directo e independiente a la verdad, de igual modo el hecho de que nuestras teorías sean exitosas es un indicio robusto para inferir que ellas son verdaderas al menos de modo aproximado. Si las teorías no fueran al menos aproximadamente verdaderas - dice el argumento- sería un milagro que ellas fueran tan exitosas. El realista, y en realidad cualquiera con sentido común, preferirá la explicación simple de que ellas son exitosas porque son verdaderas sobre la explicación milagrosa

Supongamos que le otorgamos el punto al realista y aceptamos que el éxito es una guía segura hacia la verdad. Nos encontraríamos entonces con que — dada la pluralidad de éxitos en cienciadeberíamos admitir también la posibilidad de aceptar una pluralidad de verdades. En principio, dos teorías que sean contradictorias entre sí podrían ser consideradas ambas verdaderas dado este criterio. Una objeción realista podría ser apelar al carácter aproximado de la verdad de las teorías, pero ¿qué entendemos por aproximado si incluso teorías contradictorias podrían ser ambas verdaderas? Pareciera que el concepto de aproximado se vuelve tan amplio que pierde cualquier significado relevante.

\section{Tres salidas posibles}

¿Qué hacemos para lidiar con estas múltiples verdades a las que llegamos luego de incorporar las consideraciones pluralistas en torno a la noción de éxito al NMA? Examinaremos tres salidas que surgen de la conjugación de las dos principales tesis en juego.

La primera salida consiste en defender la validez del NMA a costa de desechar las consideraciones pluralistas ofrecidas en la sección anterior. Asumir esta postura tiene la ventaja de conservar las poderosas intuiciones que motivan el realismo, a saber, que nuestras mejores teorías científicas son exitosas porque son verdaderas. Sin embargo, no estamos justificados en desechar las consideraciones pluralistas en torno a la noción de éxito sin ofrecer una cuenta alternativa que muestre de manera plausible de qué forma es posible unificar la noción de éxito en las ciencias, o

\footnotetext{
${ }^{11}$ En rigor, no sólo consideraciones epistémicas y pragmáticas toman parte en el juicio de los miembros de las comunidades científicas y filosóficas, sino también aquellas de tipo éticas, sociales, políticas, etc.
} 
Franco Bastián

Menares Paredes

bien, que muestre que las múltiples dimensiones del éxito pueden ser compatibles con la intuición realista de que sólo podemos tener una teoría verdadera para cada conjunto de fenómenos determinados.

Salvo algunas excepciones —de las que hablaremos en el siguiente párrafo- el realista está comprometido con una noción de verdad por correspondencia que exige un compromiso monista con respecto a ella. En este caso, ello significa dejar fuera la posibilidad de admitir una pluralidad de verdades dentro de un mismo dominio de investigación. Este compromiso es incompatible o con la premisa de que estamos justificados en inferir la verdad a partir del éxito de las teorías (por lo tanto con el NMA), o bien con la idea de que la actividad científica persigue una pluralidad irreductible de objetivos que se traducen en una pluralidad de concepciones de éxito científico. Si queremos conservar tanto el monismo respecto a la noción de verdad como la validez del precepto de que el éxito es conducente a la verdad, entonces debemos ofrecer una imagen que logre integrar los diferentes objetivos que mueven la actividad científica. Esta imagen, sin embargo, no ha sido desarrollada y se ve altamente improbable que pueda serlo de manera satisfactoria —dadas las consideraciones observadas en la sección anterior.

Una segunda opción es tratar de hacer compatible el NMA con el pluralismo, recaracterizando algunas de las nociones problemáticas en el asunto. Este es el camino que adoptan aquellos filósofos que se sostienen algún tipo de pluralismo realista, generalmente de la mano de un pluralismo metafísico. Chang (2017), por ejemplo, ofrece una recaracterización de inspiración pragmática de la noción de verdad, de tal manera que se hace posible y racional sostener que dos teorías que se contradicen entre sí son de igual manera ambas verdaderas. Otras instancias de pluralismo realista pueden ser encontradas en la obra de Nancy Cartwright (1999) o John Dupré (1993), quienes desarrollan su pluralismo a partir de determinada imagen metafísica de un mundo irreductible a las categorías metafísicas monistas.

El problema de seguir este segundo camino es que resulta en el planteamiento de tesis muy fuertes que resultan altamente contraintuitivas y difíciles de sostener. Pareciera que el compromiso con una noción monista de verdad estuviera profundamente arraigado en nuestra concepción de mundo y no nos es tan fácil dispensarla frente a estas consideraciones. Hay al menos dos tipos de actitudes monistas que pueden surgir respecto a este problema. Por un lado, podemos sostener que sólo podemos aceptar una teoría para cada dominio de investigación científica. Esta sería la tesis que sostendría el realista estándar, pues para él o ella las teorías científicas persiguen ofrecer descripciones verdaderas acerca del mundo y, puesto que su compromiso es con una noción monista de verdad, se deduce que no podemos tener dos teorías que versen sobre lo mismo y que sean ambas verdaderas al mismo tiempo. Una segunda actitud más débil puede ser el sostener que si bien - de acuerdo con la noción monista de verdad - no podemos sostener que existan más de una teoría verdadera para cada dominio de investigación (al menos en el caso de que estas se contradigan entre ellas), la ciencia no persigue la verdad sino que meramente la adecuación empírica de sus teorías. Por lo tanto, estamos justificados en aceptar más de una teoría para determinado dominio de investigación, pues aceptar una teoría sólo conlleva considerarla empíricamente adecuada (van Fraassen, 1980). Esta última actitud es la que probablemente adoptaría un empirista, hablaremos un poco más de ella más adelante. 
Una objeción pluralista

al argumento de los milagros

Adicionalmente al asunto sobre el monismo/pluralismo acerca de la noción de verdad, nos encontramos con la cuestión de si una concepción epistémica de la verdad es admisible dentro del marco del realismo científico. Como ya se mencionó, propuestas como la de Chang (2017) abogan por una recaracterización de la noción de verdad que es común entre las filosofías de inspiración pragmatista. En estas propuestas muchas veces se construye el concepto de «verdad» en términos fundamentalmente epistémicos. Para los y las pragmatistas postular la verdad de una proposición o teoría consiste meramente en sostener que la creencia en ella resulta útil para algún propósito relevante, o bien, que ella debe ser creída bajo condiciones epistémicas ideales. Sin embargo, como bien hace notar Anjan Chakravartty (2007, p.13), muchos realistas no se sienten cómodos con este tipo de caracterizaciones, pues consideran que asumir una teoría de la verdad por correspondencia es una condición sine qua non para cualquier clase de realismo científico. En todo caso, tal como el mismo Chakravartty (2007) propone, la cuestión de si la «verdad» deba entenderse en términos de correspondencia o de alguna otra manera es un asunto que puede quedar abierto. No parece haber argumentos conclusivos para negarle el derecho a filósofos con simpatías pragmatistas a identificarse a sí mismos como realistas ${ }^{12}$.

Una última salida al problema consiste en, simplemente, aceptar que el NMA enfrenta problemas lo suficientemente serios como para dejar de ser una alternativa atractiva para argumentar a favor del realismo. Nótese que esto no implica automáticamente la necesidad de asumir una postura antirealista respecto a la ciencia: el argumento que hemos construido sólo nos dice que el NMA enfrenta serios problemas si es llevado hasta sus últimas consecuencias, no nos dice que el realismo sea falso. El realismo puede ser defendido por otros medios, o bien puede construido de manera alternativa. El desafío, sin embargo, está planteado: ¿puede el NMA salvarse de las consecuencias indeseables a las que hemos llegado? O bien, ¿pueden los realistas ofrecer una defensa convincente de su optimismo sin acudir al NMA?

Una posible propuesta positiva frente a este último camino es la de adoptar algún tipo de pluralismo empirista. Esta postura sería empirista porque renunciaría a la tesis de que la ciencia es un asunto acerca de la verdad del mundo y en su lugar se quedaría con el requisito de la mera adecuación empírica de sus teorías. Por lo tanto, no tiene la responsabilidad de ofrecer una respuesta al argumento desarrollado en orden de salvar el NMA. En esta misma línea, la pluralidad de objetivos de las ciencias no es un problema, porque de él no se sigue que existan una pluralidad de verdades para un mismo dominio de investigación: éxito empírico es sólo eso, a saber, éxito. No tenemos por qué hacer inferencias trascendentales a partir del éxito de nuestras teorías. Sin estas inferencias no tenemos ningún problema en admitir que existan más de una teoría susceptible de ser aceptada en un mismo dominio de investigación, puesto que la convivencia de teorías exitosas no presenta ningún problema. La elección de qué teorías ocupar para los determinados objetivos que nos planteemos relativos a la ciencia pueden descansar en consideraciones de tipo pragmáticas. Tampoco es necesario ofrecer una cuenta metafísica pluralista o una reconceptualización pragmatista del concepto de verdad en orden de justificar la convivencia de teorías dentro de un

12 Nuevamente, mi gratitud al revisor anónimo que me hizo notar el punto desarrollado en este párrafo y sugerirme bibliografía pertinente. 
mismo dominio, pues estas no son consideradas como representando directamente la naturaleza del mundo ni se considera necesario que ellas satisfagan la noción de verdad en orden de ser aceptadas.

\section{Conclusión}

El pluralismo científico conlleva varias consideraciones que apuntan en dirección contraria a algunas tesis o compromisos asumidos por el realismo científico. Una de ellas es que la creencia de que estamos justificados en inferir la verdad (aproximada) de nuestras teorías a partir del éxito que ellas exhiben. Vimos que, en la práctica, no hay una noción unitaria de éxito que guíe la práctica y reflexión de la comunidad científica y filosófica. Por lo tanto, tenemos tres opciones: primero, intentar salvar la validez del NMA ofreciendo una alternativa que permita evadir las dificultades planteadas. Tal alternativa, sin embargo, no parece estar disponible en el estado actual de la discusión. En segundo lugar, podemos intentar reconceptualizar algunas de las nociones que entran en conflicto, como lo hace Chang con la noción de verdad, u ofrecer una cuenta pluralista metafísica, como lo hacen Cartwright y Dupré. Sin embargo, el lado negativo de esta alternativa radica en que descansa en premisas altamente contraintuitivas. Por último, se propone simplemente admitir que el NMA nos lleva a conclusiones indeseables y que, por lo tanto, deberíamos dispensarlo de nuestras consideraciones en torno al debate sobre el realismo científico. Frente a esto se propone asumir un empirismo pluralista que sea capaz de dar cuenta de la pluralidad de las ciencias sin comprometerse con nociones alternativas del concepto de verdad o con interpretaciones metafísicas del pluralismo.

\section{Referencias}

Borge, B. (2015). "Realismo científico hoy: a 40 años de la formulación del argumento del nomilagro." Acta Scientiarum. Human and Social Sciences 37(2): 221-233. doi: https://doi.org/gzjg

Bueno, O. (1999). "What is structural empiricism? Scientific change in an empiricist setting." Erkenntnis 50: 59-85. doi: https://doi.org/d9bbjk

Cartwright, N. (1983). How the Laws of Physics Lie. New York: Oxford University Press. doi: https://doi.org/c2xpw9

Cartwright, N. (1999). The Dappled World. Cambridge: Cambridge University Press. doi: https://doi.org/gm46

Chakravartty, A. (2007). A Metaphysics for Scientific Realism: Knowing the Unobservable. Cambridge: Cambridge University Press. doi: https://doi.org/dhf2b8

Chang, H. (2012): “Chapter 4: Active Realism and the Reality of $\mathrm{H}_{2} \mathrm{O}$." En Chang, H., Is Water $\mathrm{H}_{2} \mathrm{O}$ ? Evidence, Realism and Pluralism. Dordrecht: Springer. doi: https://doi.org/gq2b 
Una objeción pluralista

al argumento de los milagros

Chang, H. (2017): "Is pluralism compatible with scientific realism?" En Saatsi, J. (ed.) The Routledge Handbook of Scientific Realism. New York: Routledge. doi: https://doi.org/gq2c

Dupré, J. (1993). The disorder of things: Metaphysical foundations of the disunity of science. Cambridge, MA: Harvard University Press.

Glick, D. (2021). "Pluralist structural realism: The best of both worlds?", Synthese 198: 4145-4166. doi: https://doi.org/gq2d

Hacking, I. (1983). Representing and Intervening: Introductory Topics in the Philosophy of Natural Science. Cambridge: Cambridge University Press. doi: https://doi.org/gq2f

Hoyningen-Huene, P. (2013). Systematicity. The Nature of Science. Oxford: Oxford University Press. doi: https://doi.org/gq2g

Kellert, S. H., Longino, H. E. \& Waters, C. K. (2006). "Introduction: The Pluralist Stance". En Kellert, S. H., Longino, H. E. \& Waters, C. K. (eds.) Scientific Pluralism. Minneapolis: University of Minnesota Press.

Kuhn, T. (1962). The Structure of Scientific Revolutions. Chicago: University of Chicago Press. https://doi.org/g5qr

Longino, H. (1996). "Cognitive and Non-Cognitive Values in Science: Rethinking the Dichotomy." En Lynn Hankinson Nelson and Jack Nelson (eds.), Feminism, Science, and the Philosophy of Science. Dordrecht: Springer. doi: https://doi.org/df273z

Putnam, H. (1979). "What Is Mathematical Truth?" En Putnam, H., Mathematics, Matter and Method (Philosophical Papers, Vol. 1). Cambridge: Cambridge University Press. doi: https://doi.org/ccp5nq

Psillos, S. (1999). Scientific Realism: How Science Tracks Truth. London and New York: Routledge. doi: https://doi.org/gd7bk7

Psillos, S. (2011a). "The Scope and Limits of the No Miracles Argument." En Dennis Dieks, Wenceslao Gonzalo, Thomas Uebel, Stephan Hartmann \& Marcel Weber (eds.), Explanation, Prediction, and Confirmation. Dordrecht: Springer. doi: https://doi.org/fj6775

Psillos, S. (2011b). "Choosing the realist framework." Synthese 180: 301-316. doi: https://doi.org/ds88w6

Reichenbach, H. (1938). Experience and Prediction: An Analysis of the Foundations and the Structure of Knowledge. Chicago: Univ. of Chicago Press. doi: https://doi.org/d7ffkj

van Fraassen, B. C. (1980). The Scientific Image. Oxford: Clarendon Press. doi: https://doi.org/cjr6gp 
Franco Bastián

Menares Paredes

Worrall J. (2011). "The No Miracles Intuition and the No Miracles Argument." En Dennis Dieks, Wenceslao Gonzalo, Thomas Uebel, Stephan Hartmann \& Marcel Weber (eds.), Explanation, Prediction, and Confirmation. Dordrecht: Springer. doi: https://doi.org/fkhtn8 